\title{
Reply to the letter to the editor concerning: "An absorbable thread suture technique to treat snoring"
}

\author{
Jang-Woo Kwon ${ }^{1} \cdot$ Dong-Joon Park ${ }^{1}$
}

Received: 27 May 2016/Accepted: 8 June 2016/Published online: 16 June 2016

(C) Springer-Verlag Berlin Heidelberg 2016

\section{Dear Editor,}

Thank you for the opportunity to reply to the letter of Dr. Acar and Dr. Dagli to our paper "An absorbable thread suture technique to treat snoring".

To summarize the points Dr. Acar and Dr. Dagli have made, the fact that polyglactin 910's absorption period lasts approximately 60 days and its characteristics only causes minimal fibrosis and inflammation reaction will, consequently, have limited effect on shortening the length of uvula and increasing the stiffness of the soft palate.

The statements, Dr. Acar and Dr. Dagli have made, were all considered beforehand, and we have overcome the limitation by maintaining polyglactin 910 suture until complete absorption with the addition of uvula muscle overlap during suture causing muscle twisting will cause more considerable inflammation opposed to the simple suture procedure. Furthermore, nowadays, instead of one continuous suture, multiple sutures are done to create multiple overlapping of muscles leading to more twisting, and ultimately, more fibrosis and inflammation to increase the stiffness of the soft palate [1].

As for the inquiry about operation anesthesia, our premature skill mastery during the few initial procedures resulted in longer operation time under local anesthesia than we have hoped for, but after our experience slope

This reply refers to the comment available at doi:10.1007/s00405-016-4101-7.

Dong-Joon Park

rhico@yonsei.ac.kr

1 Department of Otorhinolaryngology-Head and Neck Surgery, Yonsei University Wonju College of Medicine, 20 Ilsan-ro, Wonju, Gangwon 220-701, Republic of Korea increased, there have been no difficulty in carrying out the procedure, and unless the patients prefer general anesthesia, most operations were done in local anesthesia.

As we were pointed out, the stiffness and the elevation of the soft palate were mildly relieved after post-operation, but none has returned to its original status. If symptoms were persistent, 1-2 revision surgeries were done throughout 1-2 years. However, there have been no cases, in which countless revision surgery was required in a short period of time, and most patients showed satisfaction with their result.

Again, I thank Dr. Acar and Dr. Dagli for their interest. Their remarks have made us to reconsider some facts, and we believe that the comments will help us better develop our procedure.

Sincerely,

Dong-Joon Park, MD

\section{Reference}

1. Cartmill BT, Parham DM, Strike PW, Griffiths L, Parkin B (2014) How do absorbable sutures absorb? A prospective double-blind randomized clinical study of tissue reaction to polyglactin 910 sutures in human skin. Orbit 33:437-443. doi:10.3109/01676830. 2014.950285 